\title{
Asp271 is critical for substrate interaction with the surface binding site in -agarase $A$ from Zobellia galactanivorans
}

Wilkens, Casper; Tiwari, Manish K.; Webb, Helen; Jam, Murielle; Czjzek, Mirjam; Svensson, Birte

Published in:

Proteins: Structure, Function, and Bioinformatics

Link to article, DOI:

10.1002/prot.25614

Publication date:

2019

Document Version

Peer reviewed version

Link back to DTU Orbit

Citation (APA):

Wilkens, C., Tiwari, M. K., Webb, H., Jam, M., Czjzek, M., \& Svensson, B. (2019). Asp271 is critical for substrate interaction with the surface binding site in -agarase A from Zobellia galactanivorans. Proteins: Structure, Function, and Bioinformatics, 87(1), 34-40. https://doi.org/10.1002/prot.25614

\section{General rights}

Copyright and moral rights for the publications made accessible in the public portal are retained by the authors and/or other copyright owners and it is a condition of accessing publications that users recognise and abide by the legal requirements associated with these rights.

- Users may download and print one copy of any publication from the public portal for the purpose of private study or research.

- You may not further distribute the material or use it for any profit-making activity or commercial gain

- You may freely distribute the URL identifying the publication in the public portal 
Title: Asp271 is critical for substrate interaction with the surface binding site in $\beta$-agarase A from Zobellia galactanivorans

\section{Short title: Asp271 in AgaA SBS}

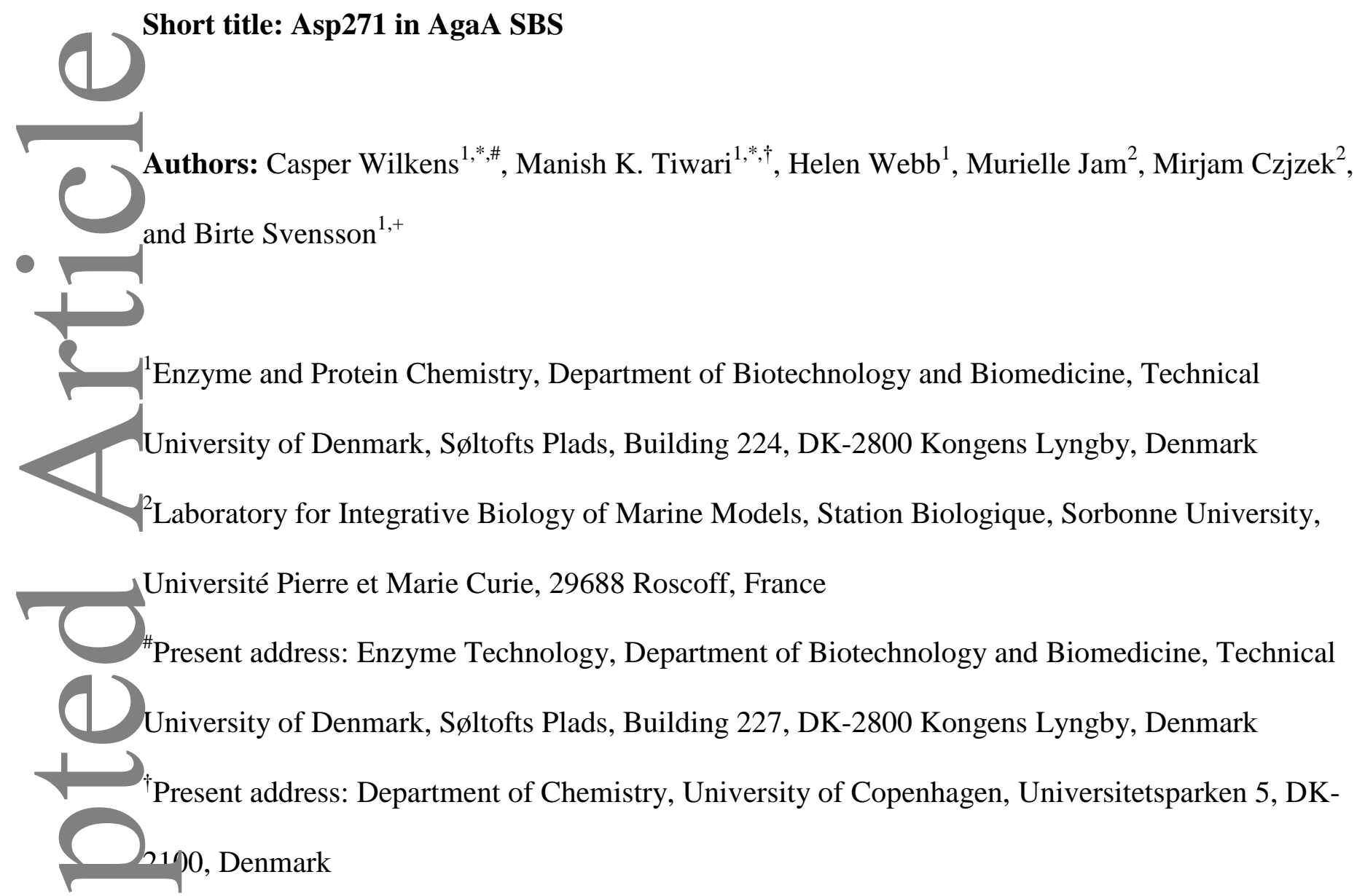

${ }^{+}$Correspondance to: B. Svensson, Enzyme and Protein Chemistry, Department of Biotechnology and Biomedicine, Technical University of Denmark, Building 224, Søltofts Plads, DK-2800 Kongens Lyngby, Denmark, Tel: +4545252740, E-mail: bis@ bio.dtu.dk

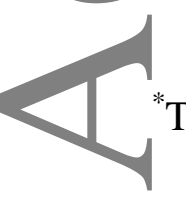

*These authors contributed equally to the work

This article has been accepted for publication and undergone full peer review but has not been through the copyediting, typesetting, pagination and proofreading process, which may lead to differences between this version and the Version of Record. Please cite this article as doi: 10.1002/prot.25614 (C) 2018 Wiley Periodicals, Inc.

Received: May 17, 2018; Revised: Nov 09, 2018; Accepted: Oct 08, 2018 
Keywords (5-10): marine bacterium; $\beta$-agarase; glycoside hydrolase family 16; surface binding site; agaro-oligosaccharides; comparative modeling; surface plasmon resonance; mutational analysis

\section{(1) Abstract}

In the marine environment agar degradation is assured by bacteria that contain large agarolytic systems with enzymes acting in various endo- and exo-modes. Agarase A (AgaA) is an endoglycoside hydrolase of family 16 considered to initiate degradation of agarose. Agarooligosaccharide binding at a unique surface binding site (SBS) in AgaA from Zobellia galactanivorans was investigated by computational methods in conjunction with a structure/sequence guided approach of site-directed mutagenesis probed by surface plasmon resonance binding analysis of agaro-oligosaccharides of DP 4-10. The crystal structure has shown that agaro-octaose interacts via H-bonds and aromatic stacking along 7 subsites ( $\mathrm{L}$ through $\mathrm{R}$ ) of the SBS in the inactive catalytic nucleophile mutant AgaA-E147S. D271 is centrally located in the extended SBS where it forms $\mathrm{H}$-bonds to galactose and 3,6-anhydrogalactose residues of agaroaose at subsites $\mathrm{O}$ and $\mathrm{P}$. We propose D271 is a key residue in ligand binding to the SBS. Thus AgaA-E147S/D271A gave slightly decreasing $K_{\mathrm{D}}$ values from $625 \pm 118$ to $468 \pm 13 \mu \mathrm{M}$ for agarohexaose, -octaose and -decaose, which represent 3-4 fold reduced affinity compared to AgaAE147S. Molecular dynamics simulations and interaction analyses of AgaA-E147S/D271A indicated disruption of an extended H-bond network supporting that D271 is critical for the functional SBS. Notably, neither AgaA-E147S/W87A nor AgaA-E147S/W277A, designed to eliminate stacking with galactose residues at subsites $\mathrm{O}$ and $\mathrm{Q}$, respectively, were produced in soluble form. W87 and W277 may thus control correct folding and structural integrity of AgaA. 


\section{Introduction}

After the discovery of a secondary binding site in pig pancreatic $\alpha$-amylase in $1966,{ }^{1}$ understanding of structural and functional implications of such surface binding sites (SBSs) has until recently progressed very slowly. ${ }^{2-5}$ Now, however, SBSs are identified in numerous crystal structures which, coupled with technical advancements in biophysical and biochemical characterization, ${ }^{2-4}$ enabled attribution of SBSs with roles in i) directing substrate into the active site, ${ }^{6-10}$ ii) disentanglement of substrate polysaccharide chains, ${ }^{6,11-14}$ iii) allosteric modulation of activity, ${ }^{15,16}$

iv) processive polysaccharide depolymerisation, ${ }^{6,13}$ and v) enzyme adherence onto storage and/or structural polysaccharides in plants and microorganisms. ${ }^{17}$ Still, details on SBSs involvement in function of glycoside hydrolases (GHs) on the molecular level are lacking behind which obviously hampers biotechnological exploitation of the SBS concept.

The marine bacterium Zobellia galactanivorans is a model organism for the increasingly recognized microbial conversion of algal biomass. ${ }^{18,19}$ The genome of Z. galactanivorans encodes four GH16 $\beta$-agarases (EC 3.2.1.81), AgaA, AgaB, AgaC, and AgaD, which cleave internal $\beta$-1,4-galactosidic linkages in agarose producing agaro-oligosaccharides (Fig. 1). ${ }^{18,20-23}$ Crystal structures are available

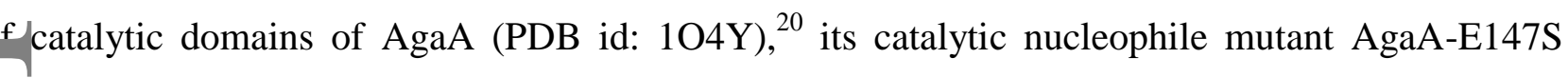
(PDB id: 1URX), ${ }^{11}$ AgaB (PDB id: 1O4Z, 4ATF) ${ }^{20,18}$ and AgaD (PDB 4id: ASM), ${ }^{18}$ but not of AgaC. AgaA and AgaB have comparable activity towards agarose gels. By contrast, AgaB is much more effective than AgaA in hydrolyzing agaro-octaose and shorter oligosaccharides. ${ }^{20}$ In the inactive mutant AgaA-E147S, the poor substrate agaro-octaose occupied 7 subsites $(\mathrm{L}-\mathrm{R})$ in an SBS running parallel to the active site cleft, which accommodates the reducing-end agaro-tetraose moiety of agaro-octaose at subsites -1 through $-4 .{ }^{20}$ Notably, agaro-decaose occupied eight subsites (L - S) in the SBS (Fig. 2). The binding to the SBS was unusual and not obtained for the $\mathrm{AgaB}$ and $\mathrm{AgaD}$ structures. AgaB thus has agaro-octaose bound at the active site, but no ligand at the area 
equivalent to the SBS in AgaA, although both enzymes appear to have a very similar concave cavity. ${ }^{11}$ Binding of agaro-octaose at the SBS in AgaA therefore appear to depend on specific amino acid residues rather than the SBS architecture. ${ }^{11}$ Notably, amino acid residues in the SBS of AgaA are not conserved among $\beta$-agarases. ${ }^{11,24}$

Based on the AgaA-E147S-agaro-octaose complex, the SBS was previously proposed to participate in unwinding double helical polysaccharide chains in agarose prior to hydrolysis of a single chain at the active site. ${ }^{11,20,22}$ So far protein engineering of $\beta$-agarase, however, has focused on improving activity and thermostability and did not explore the importance of amino acid residues in the SBS of $\operatorname{AgaA}^{25,26}$

GH16 is a large enzyme family that harbors a wide diversity of substrate specificities (www.cazy.org/). ${ }^{27}$ Expanding structural and functional insight on GH16 endo- $\beta$-agarases motivated the present investigation of the molecular interplay of agaro-oligosaccharides and the AgaA SBS. Focus is on the roles of three residues W87, D271 and W277 situated at the center of the long SBS crevice. Site-directed mutagenesis, surface plasmon resonance (SPR) analysis, molecular dynamic simulations (MD) and molecular modeling all support a key role of D271. The AgaA-E147S/D271A mutant showed reduced affinity for agaro-oligosaccharides, while the alanine (1) ubstitution of either W87 or W277, together creating a carbohydrate binding platform at the center of the SBS, hampered recombinant production of these AgaA variants suggesting a critical role also for the AgaA structural integrity.

\section{Materials and methods}

\section{Computational methods and structural setup}

The crystal structure of the inactive catalytic nucleophile mutants AgaA-E147S-agaro-octaose complex (PDB id: 1URX) ${ }^{11}$ and free AgaB-E189D (PDB id: 4ATF) ${ }^{18}$ were subjected to structure- 
based sequence alignment, mutant modeling and ligand interaction mapping using the "Prepare Protein" tools in Discovery Studio 4.0. ${ }^{28}$ Briefly, alternate conformations for five amino acid residues (M36, E54, R57, M185 and S200) in 1URX and nine amino acid residues (V65, S95, E99, T148, I159, E184, V210, H214 and V249) in 4ATF were removed and side chain conformations optimized, ${ }^{29}$ followed by hydrogen atoms being added using the CHARMm forcefield. ${ }^{30}$ To mimic physiological states and optimizing the structures for further analysis, protonation of ionizable residues at appropriate $\mathrm{pH}$ (7.0) was included applying the "Calculate protein ionization and residue pK" protocol. ${ }^{31}$

\section{Biochemical methods}

\section{Mutagenesis, heterologous expression, and purification}

pET-20b carrying the gene encoding the catalytic nucleophile mutant of the catalytic module AgaAE147S, ${ }^{22}$ was purified (GeneJet Plasmid Miniprep kit, Fermentas) from Escherichia coli DH5 $\alpha$ (Novagen) and transformed into E. coli Origami B (DE3) pLys (Novagen) by electroporation (Micropulser; Bio-Rad) followed by selection on Luria-Bertani (LB) agar plates supplemented with n') $\mu \mathrm{gl}^{-1}$ ampicillin, $15 \mu \mathrm{g} \mathrm{ml}{ }^{-1}$ kanamycin, $34 \mu \mathrm{g} \mathrm{ml}^{-1}$ chloramphenicol, and $12.5 \mu \mathrm{g} \mathrm{ml} l^{-1}$ tetracycline. The AgaA-E147S gene was subjected to site-directed mutagenesis (QuickChange kit; Stratagene) in the pET-20b vector according to the manufacturer's recommendations (primers are listed in Supplementary Table S1) and transformed into E. coli DH5 $\alpha$ followed by selection (as above). Mutations were verified by sequencing (Eurofins MWG Operon, Ebersberg, Germany) and plasmids were transformed into E. coli Origami B (DE3) pLys (as above) for heterologous expression. E. coli Origami B (DE3) pLys transformants were grown in LB medium $(4 \times 500 \mathrm{ml}$, $37^{\circ} \mathrm{C}, 18 \mathrm{~h}$ ), harvested $\left(3000 \mathrm{~g}, 10 \mathrm{~min}, 22^{\circ} \mathrm{C}\right)$, resuspended in $100 \mathrm{ml} \mathrm{M} 9$ medium (see below) and used to inoculate M9 medium (4 L) supplemented with $1 \%(\mathrm{w} / \mathrm{v})$ glucose, $2 \mathrm{ml} \mathrm{L}^{-1}$ trace elements, 
$32100 \mu \mathrm{g} \mathrm{ml}^{-1}$ ampicillin, $15 \mu \mathrm{g} \mathrm{ml}^{-1}$ kanamycin, $34 \mu \mathrm{g} \mathrm{ml} \mathrm{l}^{-1}$ chloramphenicol, and $12.5 \mu \mathrm{g} \mathrm{ml}{ }^{-1}$ tetracycline (5 L Biostat B bioreactor, B. Braun Biotech International). Glucose (50\% w/v) supplemented with $10 \mathrm{ml} \mathrm{L}^{-1}$ trace elements ${ }^{32}$ was used as carbon source during the fed-batch phase. The culture was propagated at $37{ }^{\circ} \mathrm{C}$ until $\mathrm{OD}_{600} 8-12$, followed by lowering the temperature to 20 ${ }^{\circ} \mathrm{C}$ and inducing expression by addition of isopropyl- $\beta$-D-1-thiogalactopyranoside (IPTG) to 100 $\mu \mathrm{M}$. pH 7 was maintained by addition of $28 \%$ (w/v) ammonia; temperature, foam, and dissolved oxygen tension were controlled automatically. After 16-18 h cells were harvested $(5000 \mathrm{~g}, 30 \mathrm{~min}$,

$4{ }^{\circ} \mathrm{C}$ ) at $\mathrm{OD}_{600} 16-20$ and stored at $-20{ }^{\circ} \mathrm{C}$. Cells were resuspended in $50 \mathrm{mM}$ Tris- $\mathrm{HCl}, 0.35 \mathrm{M}$ $\mathrm{NaCl}, 10 \mathrm{mM}$ imidazole $\mathrm{pH} 8$ (1/10 of the culture volume), lysed (Pressure Cell Homogeniser; Stansted Fluid Power) at $4{ }^{\circ} \mathrm{C}$, treated with benzonase nuclease (Invitrogen) $30 \mathrm{~min}$ at RT, and centrifuged $\left(20000 \mathrm{~g}, 30 \mathrm{~min}, 4^{\circ} \mathrm{C}\right)$. The supernatant was filtrated $(0.45 \mu \mathrm{m}$ Durapore membrane filters; Millipore), applied to a $1 \mathrm{ml}$ HisTrap HP column (GE Healthcare) at a flow rate of $1 \mathrm{ml} \mathrm{min}^{-}$ and eluted by a linear imidazole gradient $(0-0.5 \mathrm{M})$ in the above buffer. Fractions containing enzyme were pooled, dialyzed against $10 \mathrm{mM}$ Tris- $\mathrm{HCl} \mathrm{pH}$ 7.5, applied to $1 \mathrm{ml}$ ResourceQ column (GE Healthcare) at a flow rate of $0.5 \mathrm{ml} \mathrm{min}^{-1}$, and eluted by a linear $\mathrm{NaCl}$ gradient $(0-1 \mathrm{M})$ in 10 1 Tris-HCl pH 7.5. AgaA-E147S was prepared as described previously. ${ }^{11}$ Purity was checked by $12 \%$ SDS-PAGE and protein concentrations were determined spectrophotometrically using a theoretical molar extinction coefficient $\varepsilon_{280}=93850 \mathrm{~cm}^{-1} \mathrm{M}^{-1}{ }^{11,20}$

C)

Surface plasmon resonance

AgaA-E147S and -E147S/D271A were buffer-exchanged by dialysis into $10 \mathrm{mM}$ sodium acetate pH 4.5 and 5.5, respectively, prior to amine coupling to 1664-2056 RU (resonance units) onto CM5 chips (GE Healthcare) for SPR analysis (BIAcore T100, GE Healthcare). Sensorgrams (RU vs. time) were recorded at $25^{\circ} \mathrm{C}$ in running buffer (10 mM MOPS, $150 \mathrm{mM} \mathrm{NaCl}, 0.005 \%$ surfactant 
$\mathrm{P} 20, \mathrm{pH} 7.5$ ) at a flow rate of $10 \mu \mathrm{min}^{-1}$ with 50 or $100 \mathrm{~s}$ contact time (depending on the expected affinity) followed by $150 \mathrm{~s}$ dissociation, and baseline-corrected by subtracting data from a parallel flow cell without enzyme. $K_{\mathrm{D}}$ was calculated (eq. 1) from RU values obtained with $15 \mu \mathrm{M}-1 \mathrm{mM}$ agaro-tetraose, -hexaose, -octaose and -decaose (15 $\mu \mathrm{M}-2 \mathrm{mM}$ agaro-tetraose for AgaA-E147S) by steady-state affinity fitting (BIAcore T100 evaluation 2.0.3; GE Healthcare):

$$
R=\frac{R_{\max }[\text { Ligand }]}{[\text { Ligand }]+K_{\mathrm{D}}}(1)
$$

[Ligand] is the oligosaccharide concentration and $R_{\max }$ the maximum binding capacity in RU. The 13 stoichiometry was calculated (eq. 2):

$$
\text { stoichiometry }=\frac{R_{\max } \cdot M W_{\text {protein }}}{M W_{\text {Ligand }} \cdot R_{\text {immobilized protein }}}(2)
$$

is the protein immobilized in RU. Analyses were conducted in triplicates. $\Delta G^{\circ}$ differences were calculated (eq. 3):

$\Delta G^{\circ}=-\mathrm{RT} \log _{10} \frac{K_{D 1}}{K_{D 2}}(3)$

$K_{\mathrm{D} 1}$ and $K_{\mathrm{D} 2}$ is the dissociation constant for the highest and lowest obtained $K_{\mathrm{D}}$ for a particular agaro-oligosaccharide.

agaro-oligosaccharides were produced and purified as in Allouch et al. ${ }^{11}$

\section{(3) \\ Results and discussion}

Agaro-octaose binds to the SBS in the AgaA-E147S crystal structure facilitated by a tightly nestled H-bond network and several aromatic stacking interactions. The H-bonding involves N82, Q85, N89, Q96, Q98, and D271 (Fig. 3A) and four aromatic residues W87, Y106, Y202 and W277 participate in face-to-face and edge-to-face interactions with galactose residues in agaro-octoase (Fig. 3B). AgaA and AgaB share the same architecture at the SBS, however, binding was not observed at the area equivalent to the SBS on AgaB. The residues involved in binding the agaro- 
octaose are not conserved in AgaB (Supplemental Fig. S1), ${ }^{11}$ and the MD showed that this results in only one H-bonds being formed and steric clashes at subsite M, N, O, Q and R (Supplementary Fig. S2). Thus, binding of agaro-octaose at the SBS in AgaA seems to depend on specific amino acid residues rather than the SBS architecture.

BLAST analysis using full-length (539 amino acid residues) Z. galactanivorans AgaA (GI: 503761450 ) as query identified 30 proteins having $20.1 \%$ or higher sequence similarity from the NCBI non-redundant database. Multiple sequence alignment of these 31 GH16 homologs including Z. galactanivorans AgaA (GenBank acc. nr. WP 013995526.1) indicated W87 and W277 of the stacking aromatic residues (Fig. 3B) to be conserved as tryptophan, tyrosine or phenylalanine whereas none of the direct H-bonding residues were conserved (Supplementary Fig. S3). Mutational analysis thus comprised AgaA-E147S/W87A, -E147S/W87R, -E147S/D271A, -E147S/W277A, and -E147S/W277R. Notably, AgaA-E147S was recombinantly produced as before in rather modest amounts of about $1 \mathrm{mg} / \mathrm{L}^{22}$ and for AgaA-E147S/D271A $0.5 \mathrm{mg} / \mathrm{L}$ was obtained.

(1)

The AgaA SBS has been suggested to mediate unwinding of double helical agarose chains ${ }^{11}$ and/or dissociation of agarose fibers ${ }^{22}$ during catalysis. Here focus is turned to explicit details of (formation and possible ligand contacts of amino acid residues by applying a sequence/structure analysis approach to further investigate the AgaA SBS architecture. Unfortunately, loss of the central aromatic platform by W87A, W87R, W277A or W277R mutations probably caused severe misfolding as judged by lack of an AgaA-sized mutant band in SDS-PAGE (data not shown). Thus W87 and W277 appear critical for the conformational integrity, which was substantiated by the effect of W87 and W277 substitutions on stability calculated based on the AgaA-E147S structure ${ }^{11}$ using two web-based servers; mCSM $^{33}$ and DUET. ${ }^{34}$ Remarkably, this in silico analysis showed that replacing either W87 or W277 by any other amino acid destabilized AgaA as estimated by differences in calculated Gibbs free energy $(\Delta \Delta G$; $\mathrm{kcal} / \mathrm{mol})$ between AgaA-E147S and the mutants 
(Supplementary Tables S2 and S3). Severe destabilizing energy was predicted by both mCSM ( -2.9 $\mathrm{kcal} / \mathrm{mol})$ and DUTE $(-2.8 \mathrm{kcal} / \mathrm{mol})$ when W87 was substituted with alanine (W87A). However, when W87 was mutated to arginine (W87R), the predicated change in folding energy by mCSM (-1.3 kcal/mol) and DUTE $(-1.2 \mathrm{kcal} / \mathrm{mol})$ was less drastic (Table S2). Similar trends were observed when W277 was subjected to either alanine (W277A, $-2.9 \mathrm{kcal} / \mathrm{mol}$ ) or arginine (W227R, $-1.7 \mathrm{kcal} / \mathrm{mol}$ ) substitutions (Table S3). Cases of a tryptophan residue critical in folding have been reported for other proteins, ${ }^{35-38}$ hence loss of AgaA structural integrity may be compatible with a folding defect or mismatched hydrophobicity ${ }^{35}$ in the AgaA-E147S/W87A and AgaAE147S/W277A SBS mutants.

The importance of D271 situated in the center of the long SBS and its H-bonding with agarooctaose at subsites O and P (Fig. 3A, B) was assessed by AgaA-E147S and -E147S/D271A analysis of complex formation with agaro-tetraose, -hexaose, -octaose and -decaose using SPR and twoparameter Langmuir fit. AgaA-E147S gave $K_{\mathrm{D}}$ from $2.9 \pm 1.4 \mathrm{mM}$ for agaro-tetraose, and $K_{\mathrm{D}}$ gradually decreased with increasing oligosaccharide DP to $92.5 \pm 9.8 \mu \mathrm{M}$ for agaro-decaose (Fig. 4A; Table 1). The stoichiometry (ligand molecules bound per enzyme molecule) concomitantly decreased from 1.15 to 0.52 (Table 1). AgaA-E147S/D271A showed 3-4 fold lower affinity than AgaA-E147S for agaro-hexaose, -octaose and -decaose, but a similar decrease in stoichiometry from 1.05 to 0.69 (Table 1). Surprisingly, agaro-tetraose bound 8-fold more strongly to the D271A mutant yielding $K_{\mathrm{D}}=488 \pm 54 \mu \mathrm{M}$ (Fig. 4A; Table 1). From the structure of AgaA-E147S-agarooctaose, a stoichiometry of two is expected when the SBS and active site are both occupied. However, while $K_{\mathrm{D}}$ values were determined using ligand concentrations up to $1 \mathrm{mM}, 4 \mathrm{mM}$ agarooctaose and $10 \mathrm{mM}$ agaro-dodecaose were used for crystallization ${ }^{11}$ and these concentrations may even be higher due to evaporation during hanging-drop crystallization. It is likely therefore that the agaro-oligosaccharides are not bound at the active site in the SPR analysis as the D271A mutation 
affects affinity for all agaro-oligosaccharides (Table 1) and the SPR sensorgrams (Fig. 4B) do not show a two-state binding or dissociation as would have been expected for multiple binding sites with significantly different affinity. Notably, it is not uncommon that the SBS, as compared with the active site, has the highest affinity for the substrate. ${ }^{10,14,39,40}$

Only agaro-tetraose is visible at the active site in AgaA-E147S occupying subsites -4 through -1 (Fig. 2A), and the un-modeled tetrasaccharide part of agaro-octaose extends further towards the non-reducing end and is speculated to be required for optimal binding at the active site. ${ }^{11}$ This is in agreement with AgaA primarily releasing agaro-hexaose and -tetraose when incubated with agarose suggesting that the short agaro-tetraose, which is not a substrate, is also not accommodated in the AgaA active site. ${ }^{22}$ Thus while D271 is clearly important for binding of agaro-hexaose, -octaose and -decaose at the SBS, this is not the case for agaro-tetraose binding. By contrast, D271 seems to suppress binding, as the AgaA-D271A mutant improved agaro-tetraose interaction at the SBS. As reported in the literature the present stoichiometry above 1 indicates that two agaro-tetraose molecules can bind to the SBS in AgaA-D271A. ${ }^{3}$

A model of AgaA-E147S/D271A was calculated using AgaA-E147S as template to assess the uence of this SBS mutation on the binding mode of agaro-octaose. Firstly, to further elucidate the role of D271 and impact of D271A on the protein conformation, AgaA-E147S-agaro-octaose was superimposed with a computed structure of AgaA-E147S/D271A-agaro-octaose (see Supplementary Methods) which yielded an RMSD of $0.90 \AA$ over $268 \alpha$-carbon atoms. Notably, a positional shift of the indole ring of W87 in AgaA-E147S/D271A-agaro-octaose led to loss of stacking interaction with the D-galactopyranose ring at subsite O (Supplemental Fig. S4A). Secondly, substitution of the negatively charged and polar D271 with the smaller and non-polar alanine resulted in loss of the H-bond between D271 and subsite O (Supplemental Fig. S4B). Furthermore, changes in side chain orientations of Q96, Q98, and I275 at subsites N, O, and P were 
observed to accompany the alanine substitution at D271 (Supplemental Fig. S4A; B) and resulted in loss of two crucial H-bonds between Q96 and Q98 and the ligand at subsites O and M, respectively, in the AgaA-E147S/D271A-agaro-octaose complex. However, two new H-bonds would form between agaro-octaose and N89 at subsite Q (2.8 $)$ and K127 at subsite R (3.3 $\AA$ ) in the part of the SBS that binds the reducing-end ligand segment in AgaA-E147S/D271A (Supplemental Fig. S4B). We speculate that such reorganization contributes to the remarkable increase in affinity for agarotetraose by the $\mathrm{D} 271 \mathrm{~A}$ mutation that amounts to $\Delta G^{\circ}$ of $4.4 \mathrm{~kJ} \mathrm{~mol}^{-1}$ (Table 1). This energy difference corresponds to gain of a weak hydrogen bond for each of the proposed two bound agarotetraose molecules. For agaro-hexaose through agaro-decaose the smaller binding energy loss due to he D271A mutation is equivalent to lack of one hydrogen bond (Table 1). It is noteworthy, however, that the $\Delta G^{\circ}$ for agaro-hexaose is half of the loss for agaro-decaose (Table 1), suggesting that agaro-hexaose spans subsites $\mathrm{M}$ through $\mathrm{R}$ with the loss of a $\mathrm{H}$-bond near the reducing end to Q98 in the AgaA- E147S/D271A complex being less severe than for agaro-octaose and agarodecaose. This possibly reflects the importance of H-bonds in orientating the longer agarooligosaccharides at subsite L.

Mreover, the changes elicited by AgaA-E147S/D271A influenced ligand showing a calculated slight lateral shift in the position of the agaro-octaose at both the reducing and the non-reducing ends (Supplemental Fig. S4A). This shift in addition to loss of aromatic interactions caused agarooctaose to form only five H-bonds with the SBS in AgaA-E147S/D271A (Supplemental Fig. S4) compared to seven in AgaA-E147S (Fig 3B). At the non-reducing end of agaro-octaose bound to AgaA-E147S/D271A, N82 N82 makes two H-bond contacts, one with O3 of 3,6-anhydro-Lgalactose at subsite L, and one with $\mathrm{O} 4$ of the D-galactose at subsite M. Notably, however, compared to the AgaA-E147S-agaro-octaose complex, calculated lengths of these two H-bonds were reduced to $2.9 \AA$ (Supplemental Fig. S4B) from 3.0 and $3.2 \AA$, respectively (Fig. 3A). Despite 
this reduction in H-bond lengths in AgaA-E147S/D271A-agaro-octaose at subsite L, loss of crucial H-bonding of Q85, Q96 and Q98 with agaro-octaose at subsites $\mathrm{M}, \mathrm{N}$, and $\mathrm{O}$ in AgaAE147S/D271A due to increased distance to the carbohydrate (Supplemental Fig. S4B) may reduce the overall binding affinity, consistent with SPR results (Table 1). Towards the reducing end in AgaA-E147S/D271A-agaro-octaose, N89 interacts with O4 and O6 of D-galactopyranose at subsite Q via two H-bonds of 3.0 and $2.8 \AA$, rather than just one determined for AgaA-E147S-agarooctaose. It is remarkable that as a consequence of the D271A mutation in AgaA-E147S/D271A, the sigma- $\pi$ stacking interaction involving a centrally located D-galactopyranose ring and W87 was lost due to disruption of the hydrophobic microenvironment to which it belongs. Additionally, backbone shifting of W87, Q96, Q98, and I275, made the SBS more open in AgaA-E147S/D271A compared AgaA-E147S (Supplemental Fig. S4B). This destabilizes the interaction between residues in AgaA-E147S/D271A and agaro-octaose hence binding more loosely in agreement with the decreased affinity determined by SPR (Table 1). In previous studies, focusing on H-bond forming ability, it was suggested that perturbed H-bonds may affect substrate binding affinities, turnover, and the kinetically preferred reaction pathway. ${ }^{41-44}$ Therefore, it is not surprising that loss of H-bond Dot'work interactions by the single point mutation D271A impairs binding affinity. It is noteworthy () that only a single hypothetical protein from Aquimarina agarilytica among the 30 AgaA homologous enzymes in the multiple sequence alignment (Supplementary Fig. S3) has aspartic acid at same position as D271 (AgaB has I335), indeed suggesting unique properties of AgaA SBS. Other aligned enzymes have proline, serine or threonine at this position (Supplementary Fig. S3). Thus, D271 in AgaA is a critically positioned residue that is essential for part of the spatially extended H-bond network at the concave face of AgaA SBS and which can confer the bacterium a competitive advantage while populating its habitat niche. 


\section{Conclusion}

Collectively, the functional importance of selected SBS residues in GH16 $\beta$-agarases was disclosed using the AgaA structure (1URX) in a systematic structure/sequence based comparative analysis. The significant D271 situated at the center of the AgaA SBS and engaged in an extended H-bond network was identified and its functional importance is confirmed by an integrated biophysicalcomputational characterization indicating that mutation of D271A reduced binding affinity for longer agaro-oligosaccharides, but not for agaro-tetraose that gained affinity possibly as a consequence of the altered H-bond network. The present study outlines a plausible mechanism of how D271 participates in substrate binding at the SBS. This includes to serve as an anchor that interacts with the bound substrate and to help orienting and stabilizing the AgaA SBS structure achieving an optimized microenvironment contributing aromatic and hydrophobic interactions; a well-known feature in protein-carbohydrate complexes. Indeed, the spatially adjacent W87 and W277 create a hydrophobic cluster that seemed critical for maintaining the overall fold of AgaA. These results provide the first insight into the molecular basis for divergent substrate binding behavior in GH16 $\beta$-agarases and show that residues in the SBS of AgaA establish an important Hhothd network that can be considered in future structure-based design of GH16 $\beta$-agarases and possibly also of other structurally related GH16 enzymes.

\section{0 \\ Acknowledgments}

We are grateful to Karina Jansen for general laboratory assistance. This work was supported by The Danish Council for Independent Research | Natural Sciences (FNU) to the project 'Discovery and Characterization of Carbohydrate Surface Binding Sites (SBS) in Polysaccharide Converting Enzymes' (Grant nr. 09-027151) and to the Biacore T100 instrument (272-06-0050) (both to BS) and by $1 / 3 \mathrm{PhD}$ fellowship from the Technical University of Denmark (to $\mathrm{CW}$ ). 


\section{References}

1. Loyter A, Schramm M. Multimolecular complexes of alpha-amylase with glycogen limit dextrin. Number of binding sites of the enzyme and size of the complexes. J Biol Chem. 1966;241(11):2611-2617.

Cockburn D, Wilkens C, Ruzanski C, Andersen S, Willum Nielsen J, Smith A, Field R, Willemoës M, Abou Hachem M, Svensson B. Analysis of surface binding sites (SBSs) in carbohydrate active enzymes with focus on glycoside hydrolase families 13 and 77 - a mini-review. Biologia. 2014;69(6):705-712.

Cockburn D, Wilkens C, Dilokpimol A, Nakai H, Lewińska A, Abou Hachem M, Svensson B. Using carbohydrate interaction assays to reveal novel binding sites in carbohydrate active enzymes. PLOS ONE. 2016;11(8):e0160112.

Cuyvers S, Dornez E, Delcour JA, Courtin CM. Occurrence and functional significance of secondary carbohydrate binding sites in glycoside hydrolases. Crit Rev Biotechnol. 2012;32(2):93-107.

Samaei-Daryan S, Goliaei B, Ebrahim-Habibi A. Characterization of surface binding sites in glycoside hydrolases: A computational study. J Mol Recognit. 2017;30(9).

Nielsen MM, Bozonnet S, Seo ES, Motyan JA, Andersen JM, Dilokpimol A, Abou Hachem M, Gyemant G, Naested H, Kandra L, Sigurskjold BW, Svensson B. Two secondary carbohydrate binding sites on the surface of barley $\alpha$-amylase 1 have distinct functions and display synergy in hydrolysis of starch granules. Biochemistry. 2009;48(32):7686-7697.

Cuyvers S, Dornez E, Abou Hachem M, Svensson B, Hothorn M, Chory J, Delcour JA, Courtin CM. Isothermal titration calorimetry and surface plasmon resonance allow quantifying substrate binding to different binding sites of Bacillus subtilis xylanase. Anal Biochem. 2012;420(1):90-92.

Sevcik J, Hostinova E, Solovicova A, Gasperik J, Dauter Z, Wilson KS. Structure of the complex of a yeast glucoamylase with acarbose reveals the presence of a raw starch binding site on the catalytic domain. FEBS J. 2006;273(10):2161-2171.

Ye Z, Miyake H, Tatsumi M, Nishimura S, Nitta Y. Two additional carbohydrate-binding sites of $\beta$-amylase from Bacillus cereus var. mycoides are involved in hydrolysis and raw starch-binding. J Biochem. 2004;135(3):355-363.

Wilkens C, Cuesta-Seijo JA, Palcic M, Svensson B. Selectivity of the surface binding site (SBS) on barley starch synthase I. Biologia. 2014;69(9):1118-1121.

11. Allouch J, Helbert W, Henrissat B, Czjzek M. Parallel substrate binding sites in a $\beta$-agarase suggest a novel mode of action on double-helical agarose. Structure. 2004;12(4):623-632. 
12. Ludwiczek ML, Heller M, Kantner T, McIntosh LP. A secondary xylan-binding site enhances the catalytic activity of a single-domain family 11 glycoside hydrolase. J Mol Biol. 2007;373(2):337-354.

13. Nielsen JW, Kramhoft B, Bozonnet S, Abou Hachem M, Stipp SL, Svensson B, Willemoes M. Degradation of the starch components amylopectin and amylose by barley $\alpha$-amylase 1 : role of surface binding site 2. Arch Biochem Biophys. 2012;528(1):1-6.

14. Wilkens C, Auger KD, Anderson NT, Meekins DA, Raththagala M, Abou Hachem M, Payne CM, Gentry MS, Svensson B. Plant $\alpha$-glucan phosphatases SEX4 and LSF2 display different affinity for amylopectin and amylose. FEBS Letters. 2016;590(1):118-128.

15. Skov LK, Mirza O, Sprogoe D, Dar I, Remaud-Simeon M, Albenne C, Monsan P, Gajhede M. Oligosaccharide and sucrose complexes of amylosucrase. Structural implications for the polymerase activity. J Biol Chem. 2002;277(49):47741-47747.

Wilkens C, Andersen S, Petersen BO, Li A, Busse-Wicher M, Birch J, Cockburn D, Nakai H, Christensen HE, Kragelund BB, Dupree P, McCleary B, Hindsgaul O, Hachem MA, Svensson B. An efficient arabinoxylan-debranching $\alpha$-L-arabinofuranosidase of family GH62 from Aspergillus nidulans contains a secondary carbohydrate binding site. Appl Microbiol Biotechnol. 2016;100(14):6265-6277.

17. Patrick WM, Nakatani Y, Cutfield SM, Sharpe ML, Ramsay RJ, Cutfield JF. Carbohydrate binding sites in Candida albicans exo- $\beta$-1,3-glucanase and the role of the Phe-Phe 'clamp' at the active site entrance. FEBS J. 2010;277(21):4549-4561.

Hehemann JH, Correc G, Thomas F, Bernard T, Barbeyron T, Jam M, Helbert W, Michel G, Czjzek M. Biochemical and structural characterization of the complex agarolytic enzyme system from the marine bacterium Zobellia galactanivorans. $\mathrm{J}$ Biol Chem. 2012;287(36):30571-30584.

Barbeyron T, Thomas F, Barbe V, Teeling H, Schenowitz C, Dossat C, Goesmann A, Leblanc C, Oliver Glöckner F, Czjzek M, Amann R, Michel G. Habitat and taxon as driving forces of carbohydrate catabolism in marine heterotrophic bacteria: example of the model algae-associated bacterium Zobellia galactanivorans Dsij ${ }^{\mathrm{T}}$. Environ Microbiol. 2016;18(12):4610-4627.

Allouch J, Jam M, Helbert W, Barbeyron T, Kloareg B, Henrissat B, Czjzek M. The threedimensional structures of two $\beta$-agarases. J Biol Chem. 2003;278(47):47171-47180.

Hehemann JH, Michel G, Barbeyron T, Czjzek M. Expression, purification and preliminary $\mathrm{X}$-ray diffraction analysis of the catalytic module of a $\beta$-agarase from the flavobacterium Zobellia galactanivorans. Acta Crystallogr Sect F Struct Biol Cryst Commun. 2010;66(Pt 4):413-417.

22. Jam M, Flament D, Allouch J, Potin P, Thion L, Kloareg B, Czjzek M, Helbert W, Michel G, Barbeyron T. The endo- $\beta$-agarases AgaA and AgaB from the marine bacterium Zobellia galactanivorans: two paralogue enzymes with different molecular organizations and catalytic behaviours. Biochem J. 2005;385(Pt 3):703-713. 
23. Hehemann J-H, Boraston AB, Czjzek M. A sweet new wave: structures and mechanisms of enzymes that digest polysaccharides from marine algae. Curr Opin Struct Biol. 2014;28:7786.

24. Takagi E, Hatada Y, Akita M, Ohta Y, Yokoi G, Miyazaki T, Nishikawa A, Tonozuka T. Crystal structure of the catalytic domain of a GH16 $\beta$-agarase from a deep-sea bacterium, Microbulbifer thermotolerans JAMB-A94. Biosci Biotechnol Biochem. 2015;79(4):625632.

Lee S, Lee DG, Jang MK, Jeon MJ, Jang HJ, Lee SH. Improvement in the catalytic activity of $\beta$-agarase AgaA from Zobellia galactanivorans by site-directed mutagenesis. J Microbiol Biotechnol. 2011;21(11):1116-1122.

Jang MK, Lee SW, Lee DG, Kim NY, Yu KH, Jang HJ, Kim S, Kim A, Lee SH. Enhancement of the thermostability of a recombinant $\beta$-agarase, AgaB, from Zobellia galactanivorans by random mutagenesis. Biotechnol Lett. 2010;32(7):943-949.

Lombard V, Golaconda Ramulu H, Drula E, Coutinho PM, Henrissat B. The carbohydrateactive enzymes database (CAZy) in 2013. Nucleic Acids Res. 2014;42(Database issue):D490-495.

Dassault Systèmes BIOVIA, Discovery Studio, 4.0, San Diego, Dassault Systèmes, (2015).

Tiwari MK, Kepp KP. Pathogenic properties of Alzheimer's $\beta$-amyloid identified from structure-property patient-phenotype correlations. Dalton Trans. 2015;44(6):2747-2754.

Brooks BR, Brooks CL, 3rd, Mackerell AD, Jr., Nilsson L, Petrella RJ, Roux B, Won Y, Archontis G, Bartels C, Boresch S, Caflisch A, Caves L, Cui Q, Dinner AR, Feig M, Fischer S, Gao J, Hodoscek M, Im W, Kuczera K, Lazaridis T, Ma J, Ovchinnikov V, Paci E, Pastor RW, Post CB, Pu JZ, Schaefer M, Tidor B, Venable RM, Woodcock HL, Wu X, Yang W, York DM, Karplus M. CHARMM: the biomolecular simulation program. J Comput Chem. 2009;30(10):1545-1614.

Spassov VZ, Yan L. A fast and accurate computational approach to protein ionization. Protein Sci. 2008;17(11):1955-1970.

32. Holme T, Arvidson S, Lindholm B, Pavlu B. Enzymes - laboratory-scale production. Process Biochem. 1970;5:62-66.

Pires DEV, Ascher DB, Blundell TL. mCSM: predicting the effects of mutations in proteins using graph-based signatures. Bioinformatics. 2014;30(3):335-342.

Pires DEV, Ascher DB, Blundell TL. DUET: a server for predicting effects of mutations on protein stability using an integrated computational approach. Nucl Acids Res. 2014;42(W1):W314-W319.

35. de Jesus AJ, Allen TW. The role of tryptophan side chains in membrane protein anchoring and hydrophobic mismatch. Biochim Biophys Acta. 2013;1828(2):864-876. 
36. Wang QY, Manicassamy B, Yu X, Dolmer K, Gettins PG, Rong L. Characterization of the LDL-A module mutants of Tva, the subgroup A Rous sarcoma virus receptor, and the implications in protein folding. Protein Sci. 2002;11(11):2596-2605.

37. Matthews BW. Hydrophobic interactions in proteins. eLS: John Wiley \& Sons, Ltd; 2001.

38. Sanchez KM, Gable JE, Schlamadinger DE, Kim JE. Effects of tryptophan microenvironment, soluble domain, and vesicle size on the thermodynamics of membrane protein folding: lessons from the transmembrane Protein OmpA. Biochemistry. 2008;47(48):12844-12852.

Kasvinsky PJ, Madsen NB, Fletterick RJ, Sygusch J. X-ray crystallographic and kinetic studies of oligosaccharide binding to phosphorylase. J Biol Chem. 1978;253(4):1290-1296.

Larner J, Takeda Y, Hizukuri S. The influence of chain size and molecular weight on the kinetic constants for the span glucose to polysaccharide for rabbit muscle glycogen synthase. Mol Cell Biochem. 1976;12(3):131-136.

Kiefer LL, Paterno SA, Fierke CA. Hydrogen bond network in the metal binding site of carbonic anhydrase enhances zinc affinity and catalytic efficiency. J Am Chem Soc. 1995;117(26):6831-6837.

42. Hammes-Schiffer S, Benkovic SJ. Relating protein motion to catalysis. Annu Rev Biochem. 2006;75:519-541.

43. Miller GP, Benkovic SJ. Strength of an interloop hydrogen bond determines the kinetic pathway in catalysis by Escherichia coli dihydrofolate reductase. Biochemistry. 1998;37(18):6336-6342.

44. Tiwari MK, Kalia VC, Kang YC, Lee J-K. Role of a remote leucine residue in the catalytic function of polyol dehydrogenase. Mol BioSyst. 2014;10(12):3255-3263. 
AgaB occupy the active site and no ligand binds to the concave surface binding site area. Subsites of the active site are labelled. (B) Close up of the SBS cavity of both AgaA and AgaB with agarooctaose bound to AgaA SBS. Figures were generated using DS4.0 visualizer.

Fig. 3. Analysis of the agaro-octaose interactions in the SBS of the AgaA. (A) Interactions of AgaA SBS amino acid residues within $5 \AA$ area of the bound agaro-octaose in the SBS. (B) Aromatic interactions of AgaA SBS amino acid residues within $5 \AA$ area of the bound agaro-octaose in the SBS. AgaA residues are shown with off-white color. Agaro-octaose in the SBS is shown in yellow color carbon. All the amino acid residues and bound ligand are in stick model and numbering is according to their PDB structure (PDB ID 1URX). Conventional H-bonds are shown with dotted green lines and distances of these interactions are in $\AA$. Blue surface represents edge-toedge whereas orange surface shows edge-to-face aromatic interactions.

Fig. 4. Binding of AgaA-E147S and -E147S-D271A to four agaro-oligosaccharides as investigated using surface plasmon resonance. (A) Binding of AgaA-E147S with agaro-tetraose (1) ), agaro-hexaose $(\bullet)$, agaro-octaose (๘), and agaro-decaose ( $\bullet$ ), and AgaA-E147S/D271A with agaro-tetraose $(\Delta)$, agaro-hexaose $(\circ)$, agaro-octaose $(\square)$, and agaro-decaose $(\diamond)$ in triplicates. B) Representative sensorgrams for AgaA-E147S with $500 \mu \mathrm{M}$ agaro-tetraose (red), agaro-hexaose (green), agaro-octaose (blue), and agaro-decaose (black), and AgaA-E147S/D271A with $500 \mu \mathrm{M}$ agaro-tetraose (dark red), agaro-hexaose (dark green), agaro-octaose (dark blue), and agaro-decaose (grey). Association time were 50 or $100 \mathrm{~s}$ and dissociation was $150 \mathrm{~s}$ (not shown for all ligand concentrations). 


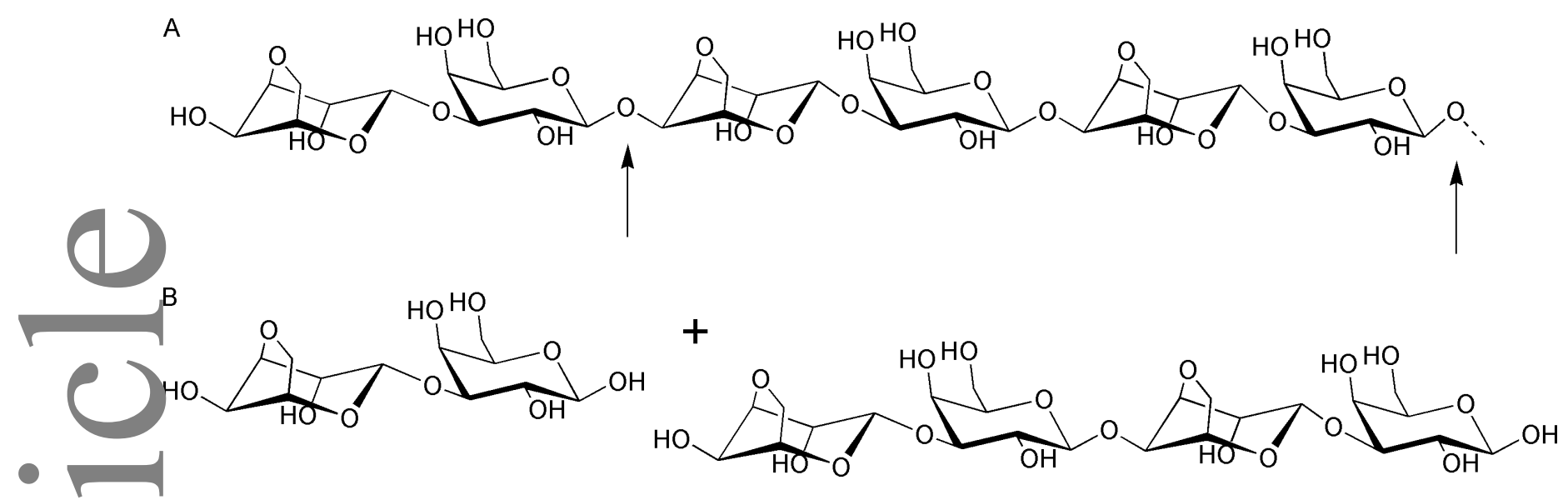




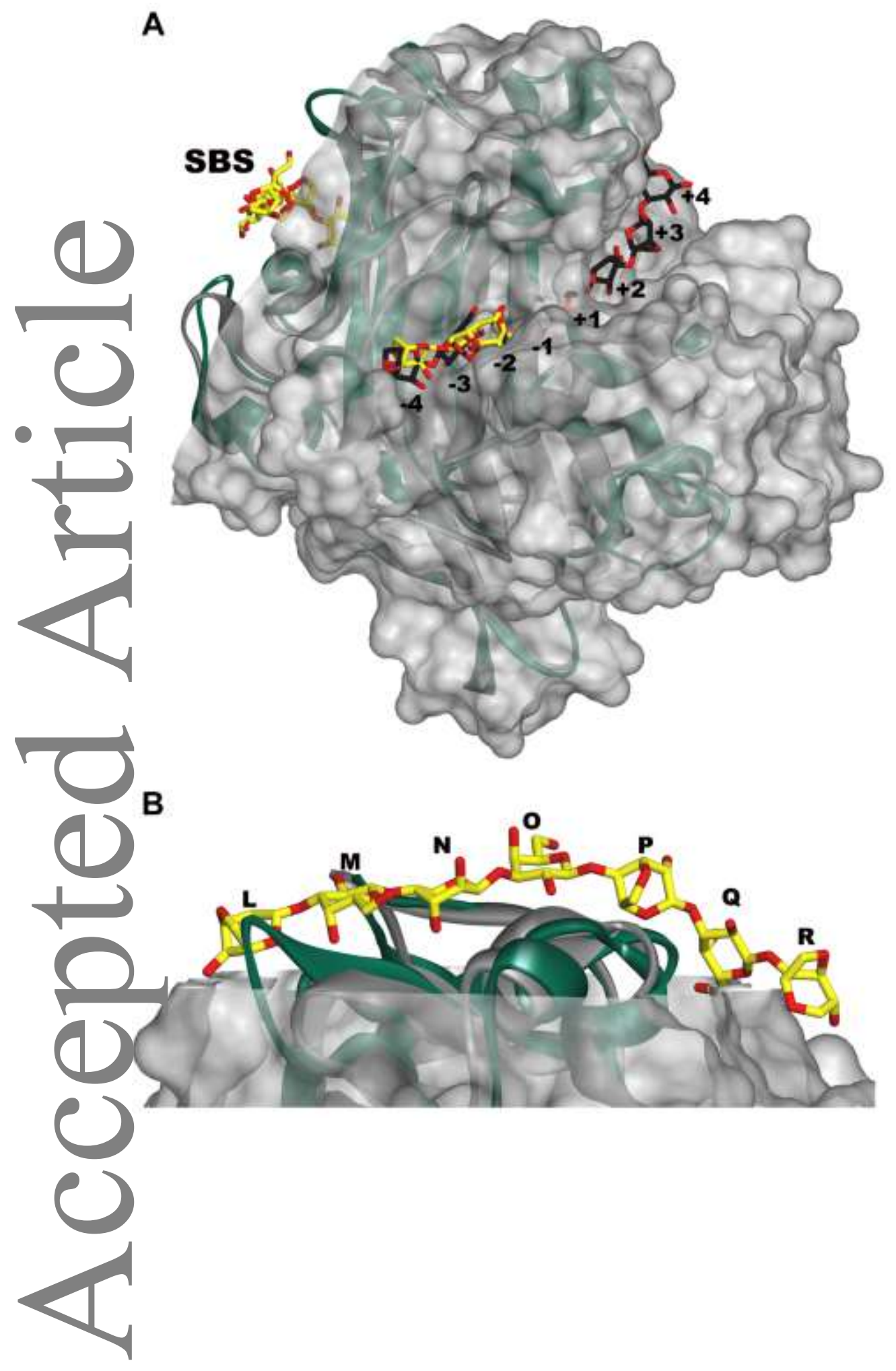




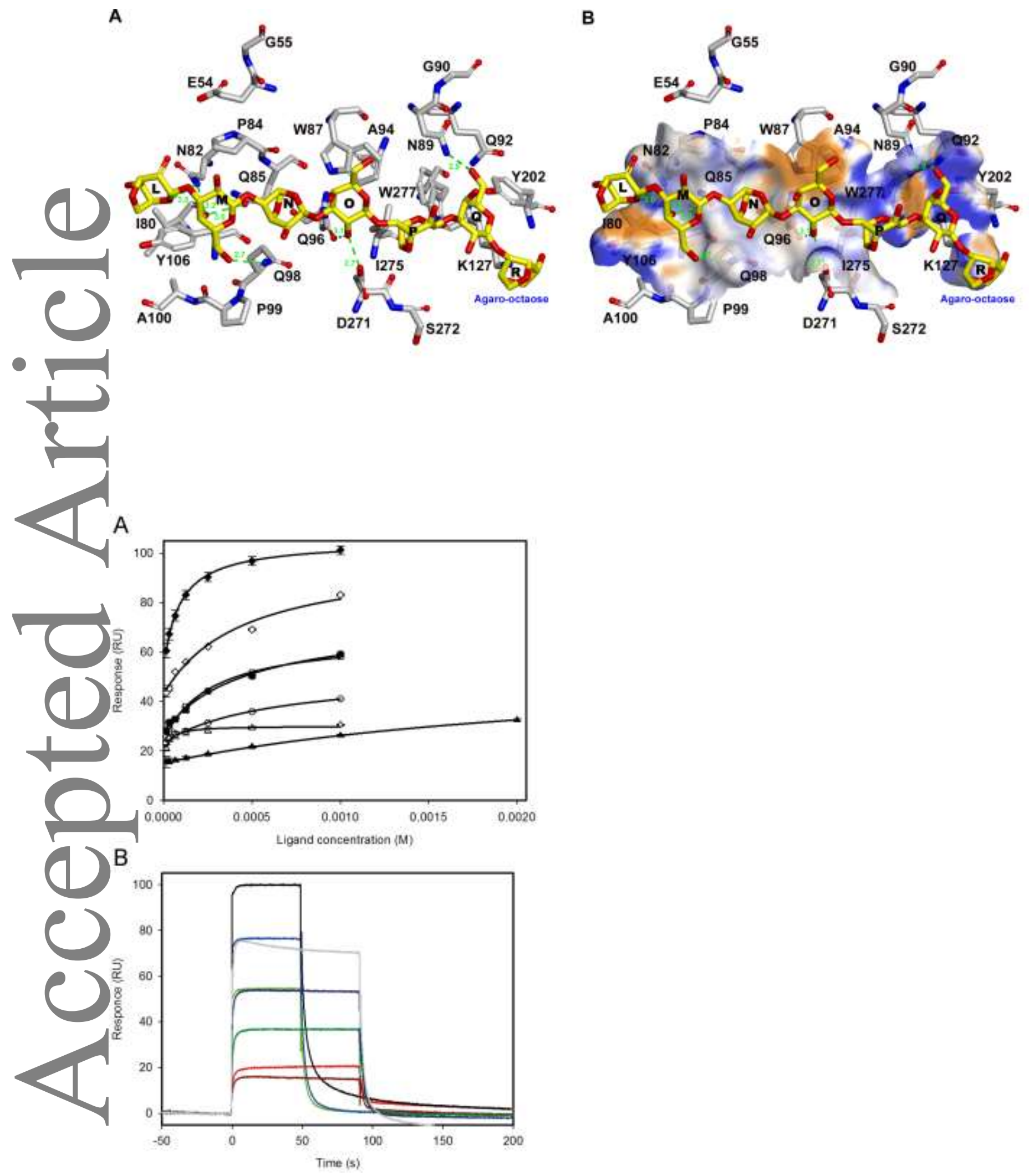

